\title{
Padronização de uma técnica de congelamento de sêmen em cães
}

\author{
Standartization of a technique for canine semen freezing \\ Mariana Machado Neves ${ }^{1}$, Marc Henry', \\ Carlos Augusto Alanis Clemente' \& Luiz Guilherme Dias Heneine ${ }^{2}$
}

\begin{abstract}
RESUMO
Várias pesquisas têm sido desenvolvidas com o intuito de melhorar a técnica da criopreservação de sêmen em cães. O objetivo deste trabalho foi estabelecer o meio diluidor de congelamento do sêmen canino mais adequado para um protocolo de congelamento realizado em máquina computadorizada, além de otimizar a temperatura de descongelamento. Foram usados cinco cães e coletados três ejaculados/animal. Foram testados os meios Tris-cítrico (G1), Lactose-gema (G2) e INRA 82 (G3) com 5\% de glicerol. Alíquotas de sêmen foram diluídas em cada meio e foram congeladas em máquina computadorizada programada para realizar uma curva de resfriamento de $-0,5^{\circ} \mathrm{C} / \mathrm{min}$, de $25-29^{\circ} \mathrm{C}$ até $5^{\circ} \mathrm{C}$, período de equilíbrio de $1 \mathrm{~h}$ a $5^{\circ} \mathrm{C}$, seguida da curva de congelamento de $-20^{\circ} \mathrm{C} / \mathrm{min}$, de $5^{\circ} \mathrm{C}$ até $-120^{\circ} \mathrm{C}$. Foram testadas duas temperaturas de descongelamento: A) $37^{\circ} \mathrm{C} / 30 \mathrm{~s}$ e B) $52^{\circ} \mathrm{C} / 10 \mathrm{~s}$, seguida de $37^{\circ} \mathrm{C} / 30 \mathrm{~s}$. Os espermatozóides foram avaliados quanto à motilidade progressiva, morfologia, reatividade ao teste hiposmótico e integridade de membranas avaliadas pelo uso de fluorescência (CFDA/IP). Os meios de congelamento Tris-cítrico e Lactose-gema foram superiores ao meio INRA quanto aos parâmetros de motilidade e morfologia espermática $(\mathrm{p}<0,05)$. Não houve diferença entre eles quanto à manutenção da integridade funcional e estrutural das membranas espermáticas ( $\mathrm{p}>0,05)$. $\mathrm{O}$ descongelamento a $52^{\circ} \mathrm{C}$ resultou no aparecimento de maior porcentagem de anormalidades acrossomais que o descongelamento a $37^{\circ} \mathrm{C}(\mathrm{p}<0,05)$. Com base nos resultados, pode-se concluir que os meios Triscítrico e Lactose-gema preservaram melhor a viabilidade espermática após o descongelamento a $37^{\circ} \mathrm{C} / 30 \mathrm{~s}$ na curva de resfriamento de $-0,5^{\circ} \mathrm{C} / \mathrm{min}$ e de congelamento de $-20^{\circ} \mathrm{C} / \mathrm{min}$.
\end{abstract}

Descritores: sêmen canino, congelamento, meio diluidor, temperatura de descongelamento.

\section{ABSTRACT}

Many researches have been developed to improve the canine semen cryopreservation technique. The aims of the present work were to determine the freezing extenders adequacy to freeze canine semen using a computadorized system. Two thaw temperature was evaluated as well. Five dogs were used and three ejaculates/animal were collected. The extenders tested were Tris-citric-yolk (G1), lactose-yolk (G2) and INRA 82 (G3), with 5\% glycerol. A computadorized freezing system was used and cooling rate was $-0,5^{\circ} \mathrm{C} / \mathrm{min}$, from $25-29^{\circ} \mathrm{C}$ to $5^{\circ} \mathrm{C}$, the equilibration period was at $5^{\circ} \mathrm{C} / 1 \mathrm{~h}$ followed by freezing the samples at a rate of $-20^{\circ} \mathrm{C} / \mathrm{min}$, from $5^{\circ} \mathrm{C}$ to $-120^{\circ} \mathrm{C}$. Thawing temperatures were: A) $37^{\circ} \mathrm{C} / 30 \mathrm{~s}$ and $\left.\mathrm{B}\right) 52^{\circ} \mathrm{C} / 10 \mathrm{~s}$ followed by $37^{\circ} \mathrm{C} / 30 \mathrm{~s}$. Evaluation post thaw attributes were spermatic motility and morphology, HOST and sperm membrane integrity by a fluorescence test using CFDA/PI. Motility and sperm morphology were better preserved with the extenders Tris-citric yolk and lactose-yolk than with INRA $82(\mathrm{p}<0.05)$. No difference was observed among treatments as for CFDA/PI and HOST $(\mathrm{p}>0.05)$ evaluations. Thawed temperature of $52^{\circ} \mathrm{C}$ provoked more acrosome abnormality than when $37^{\circ} \mathrm{C}$ was used $(\mathrm{p}<0.05)$. In conclusions, Triscitric and Lactose-yolk were more efficient than INRA 82 to preserve the spermatozoa viability post-thaw and thawing at $37^{\circ} \mathrm{C} / 30$ s showed to be more suitable when the cooling and freezing curve were $-0,5^{\circ} \mathrm{C} / \mathrm{min}-20^{\circ} \mathrm{C} / \mathrm{min}$, respectively.

Keywords: canine semen, freezing extender, thawed temperature.

Trabalho originado da Tese do primeiro autor. Programa de Pós-graduação em Ciência Animal (PPCA), Escola de Veterinária da Universidade Federal de Minas Gerais (UFMG), Av. Antonio Carlos, 6627, C.P. 567, Campus da UFMG, CEP 31270-901 Belo Horizonte, MG, Brasil. ${ }^{1}$ Departamento de Clínica e Cirurgia Veterinárias, Escola de Veterinária, UFMG. ${ }^{2}$ Departamento de Pesquisa e Desenvolvimento, Fundação Ezequiel Dias, Belo Horizonte, MG. Órgão financiador: FAPEMIG. CORRESPONDÊNCIA: M. Henry [henrym@ufmg.br ; Fax: +55 (31) 3409 2230]. 


\section{INTRODUÇÃO}

O sucesso da técnica de congelamento é expresso pela viabilidade espermática após o descongelamento e depende de fatores inerentes aos animais, como as diferenças entre raças e indivíduos, ligadas à qualidade seminal e resistência espermática a baixas temperaturas e à definição das etapas utilizadas durante o processo de criopreservação e descongelamento [6]. Neste aspecto, para a padronização de uma curva de resfriamento e de congelamento, é necessário eliminar os efeitos do local onde elas são executadas, do meio diluidor usado e da temperatura de descongelamento $[8,11,23]$.

A literatura cita o uso de caixa de isopor com gelo, geladeira e até freezer a $-152^{\circ} \mathrm{C}$ para promover ambientes de baixa temperatura durante a criopreservação $[1,2,4]$. São poucos os trabalhos com sêmen canino que utilizam máquina computadorizada capaz de realizar as curvas de resfriamento e de congelamento. A vantagem deste aparelho é a garantia da obtenção de curvas com queda homogênea e gradual da temperatura até $-120^{\circ} \mathrm{C}$, além de permitir a programação da velocidade desejada [5].

Dentre os meios diluidores existentes, o Triscítrico-gema é o meio padrão para o congelamento do sêmen canino, sendo que o meio lactose-gema vem apresentando resultados satisfatórios pós-descongelamento, provavelmente devido ao seu efeito osmótico adicional $[12,13]$. O INRA 82 é um meio diluidor formulado para equinos que ainda não foi testado em cães [21].

O objetivo deste trabalho é estabelecer o meio diluidor e a temperatura de descongelamento mais adequados para serem utilizados em curvas de resfriamento e congelamento pré-determinadas e executadas por uma máquina computadorizada.

\section{MATERIAS E MÉTODOS}

\section{Coleta do sêmen}

Foram utilizados cinco cães, dois da raça Sharpei e três da raça Labrador, com idades entre 2 e 5 anos. Após o esgotamento das reservas espermáticas, foram coletados três ejaculados de cada animal, com intervalos de 72 horas entre as coletas, totalizando 15 ejaculados.

\section{Protocolo de congelamento e avaliações espermáticas}

O sêmen fresco foi dividido em três alíquotas de igual volume. Cada alíquota foi diluída no meio de centrifugação Glicose-EDTA [10] (pH 6,7; 429mOsm/L) na proporção de 1:3 e centrifugada a $755 x$ g por sete minutos. Cada pellet foi ressuspendido no meio de congelamento em teste: T1 (Controle): Tris-cítrico com 20\% de gema do ovo e $5 \%$ de glicerol $(\mathrm{pH} 6,8 ; 885 \mathrm{mOsm} / \mathrm{L})$; T2: Lactose-gema [10] com $20 \%$ de gema do ovo e $5 \%$ de glicerol ( $\mathrm{pH} 6,7 ; 1214 \mathrm{mOsm} / \mathrm{L})$; T3: INRA 82 [14] com $2 \%$ de gema do ovo e $5 \%$ de glicerol ( $\mathrm{pH}$ $6,8 ; 1125 \mathrm{mOsm} / \mathrm{L})$.

Cada tratamento teve seu volume diluído para obtenção final de 100 milhões de espermatozóides $/ \mathrm{mL}$ e envase em palhetas de $0,5 \mathrm{~mL}$. As palhetas foram imediatamente colocadas na máquina (Modelo TK3000 - Tetakon, Brasil) programada para resfriá-las a $-0,5^{\circ} \mathrm{C} / \mathrm{min}$, da temperatura ambiente $\left(\sim 25^{\circ} \mathrm{C}\right)$ até $5^{\circ} \mathrm{C}$. Nesta temperatura, foram mantidas em equilíbrio por 1 hora. A taxa de resfriamento entre $5^{\circ} \mathrm{C} \mathrm{e}-120^{\circ} \mathrm{C}$ foi de $-20^{\circ} \mathrm{C} / \mathrm{min}$. Atingida esta temperatura, as palhetas foram submersas e armazenadas em nitrogênio líquido até o descongelamento. No descongelamento, foram testadas as temperaturas de (A) $37^{\circ} \mathrm{C}$ por 30 segundos e de (B) $52^{\circ} \mathrm{C}$ por 10 segundos, seguida de $37^{\circ} \mathrm{C}$ por 30 segundos.

A motilidade progressiva foi estimada por três avaliadores experientes utilizando-se microscopia óptica entre lâmina e lamínula aquecida. Os valores apresentados correspondem à média aritmética dos resultados obtidos pelos avaliadores. A morfologia espermática foi avaliada utilizando-se microscopia de contraste de fase em amostras coletadas imediatamente após a coleta e após o descongelamento. Após o descongelamento, os espermatozóides foram submetidos ao teste hiposmótico usando uma solução de frutose a $60 \mathrm{mOsmol} / \mathrm{L}$ [9] e a integridade de membranas foi avaliada pela coloração utilizando os fluorocromos diacetato de carboxifluoresceina (CFDA) e iodeto de propídio (IP), segundo a técnica descrita por [7] e modificada por [24]. Após o descongelamento, a motilidade progressiva foi avaliada a cada 30 minutos, até a mesma atingir 5\%.

\section{Análise estatística}

O delineamento experimental foi blocos ao acaso com parcelas subdivididas, eliminando-se o efeito cão e testando-se os meios diluidores (parcelas) descongelados em duas temperaturas (subparcelas). Os resultados foram submetidos a ANOVA, comparando-se as médias pelo teste de Student Newman Keuls (5\%) para os meios de congelamento e teste de Duncan (5\%) para as temperaturas de descongelamento. As análises estatísticas foram realizadas utilizando-se o programa estatístico SAS (Statistical Analysis System). 


\section{RESULTADOS}

A motilidade progressiva dos espermatozóides congelados nos meios Tris-cítrico-gema e lactose-gema foi melhor que a do meio INRA $82(\mathrm{p}<0,05)$, tanto após o descongelamento (Tabela 1) quanto durante o teste de termorresistência, independentemente da temperatura de descongelamento ( $>0,05$ ). Não houve diferença nas taxas de queda da motilidade progressiva entre os meios diluidores quando descongelados a $37^{\circ} \mathrm{C} / 30$ s ou $52^{\circ} \mathrm{C} / 10$ s seguido de $37^{\circ} \mathrm{C} / 30$ s.

A média de espermatozóides morfologicamente normais foi maior nos tratamentos com Triscítrico e lactose-gema, diferentemente do obtido para o meio INRA $82(\mathrm{p}<0,05)$ (Tabela 2), independente da temperatura de descongelamento utilizada. O INRA 82 apresentou maior média de defeitos de peça intermediária $(18 \pm 9,2 \%)$ que o Tris-cítrico $(4 \pm 1,6 \%)$ e a Lactosegema $(5 \pm 2,5 \%)$. Já o descongelamento a $52^{\circ} \mathrm{C}$, independentemente do meio diluidor utilizado, apresentou médias superiores de defeitos de acrossoma $(4 \pm 1,5 \%)$ e de peça intermediária $(10 \pm 7,7 \%)$ que o descongelamento a $37^{\circ} \mathrm{C}(2 \pm 3 \%$ e $7,5 \pm 2,3 \%$, respectivamente).

A reatividade ao teste hiposmótico foi semelhante entre os meios de congelamento testados (Tabela 2), independentemente da temperatura de descongelamento utilizada ( $>00,05)$, assim como a preservação da integridade funcional das membranas plasmática e acrossomal $(p>0,05)$.

\section{DISCUSSÃO}

Vários fatores podem explicar os resultados obtidos pelos meios Tris-citrico-gema e lactose-gema na preservação de parâmetros espermáticos após o congelamento em máquina computadorizada programada. Ambos possuem monossacarídeos, frutose e glicose, respectivamente, que servem como combustível energético para a manutenção da motilidade espermática. Os monossacarídeos também exercem um papel importante na preservação da morfologia espermática e da integridade das membranas, verificadas pelos testes de fluorescência e hiposmótico, ao possuir efeito osmótico e contribuir na diminuição do ponto crioscópico da água [17]. O dissacarídeo lactose do meio lactose-gema exerce um efeito osmótico ao não ultrapassar a membrana espermática, o que favorece a osmolaridade do meio extracelular para a preservação da integridade de membrana [15].

O INRA 82 também conseguiu manter a integridade funcional e estrutural da membrana plasmática,

Tabela 1. Motilidade progressiva de espermatozóides caninos congelados em três meios diluidores e descongelados em duas temperaturas, utilizando-se uma máquina computadorizada com curvas de resfriamento $\left(-0.5^{\circ} \mathrm{C} / \mathrm{min}\right)$ e congelamento $\left(-20^{\circ} \mathrm{C} / \mathrm{min}\right)$ programadas.

\begin{tabular}{ccc}
\hline \multirow{2}{*}{ Tratamento } & \multicolumn{2}{c}{ Motilidade progressiva (\%) } \\
\cline { 2 - 3 } & A & B \\
\hline G1 & $42,7 \pm 17,7^{\mathrm{a}}$ & $42 \pm 14,9^{\mathrm{a}}$ \\
G2 & $44,3 \pm 13,1^{\mathrm{a}}$ & $38 \pm 5,1^{\mathrm{a}}$ \\
G3 & $15,7 \pm 8,9^{\mathrm{b}}$ & $20 \pm 14,9^{\mathrm{b}}$ \\
\hline
\end{tabular}

$\overline{\text { Média } \pm \text { desvio-padrão; a,b - Valores com sobrescritos dife- }}$ rentes na mesma coluna são estatisticamente diferentes de acordo com o teste de Student Newman Keuls para os meios diluidores $(\mathrm{p}<0,05)$; Não houve diferença significativa na motilidade entre temperaturas de descongelamento para os três meios testados. G1: Tris-cítrico-gema; G2: Lactose-gema; G3: INRA $82 ; \mathrm{A}: 37^{\circ} \mathrm{C} / 30 \mathrm{~s} ; \mathrm{B}: 52^{\circ} \mathrm{C} / 10 \mathrm{~s}$ seguido de $37^{\circ} \mathrm{C} / 30 \mathrm{~s}$.

Tabela 2. Frequência de espermatozóides caninos morfologicamente normais, reativos ao teste hiposmótico (HO) e com a estrutura das membranas íntegras avaliadas pelo CFDA/IP congelados em três meios diluidores e descongelados em duas temperaturas, utilizando-se uma máquina computadorizada com curvas de resfriamento $\left(-0.5^{\circ} \mathrm{C} / \mathrm{min}\right)$ e congelamento $\left(-20^{\circ} \mathrm{C} / \mathrm{min}\right)$ programadas.

\begin{tabular}{ccccccc}
\hline \multirow{2}{*}{ Tratamentos } & \multicolumn{2}{c}{ Normais (\%) } & \multicolumn{2}{c}{ HO (\%) } & \multicolumn{2}{c}{ Íntegros (\%) } \\
\cline { 2 - 7 } & A & B & A & B & A & B \\
\hline G1 & $56 \pm 9,1^{\mathrm{a}}$ & $58 \pm 14,8^{\mathrm{a}}$ & $53 \pm 3,5^{\mathrm{a}}$ & $56 \pm 4,8^{\mathrm{a}}$ & $45 \pm 6,2^{\mathrm{a}}$ & $48 \pm 11,3^{\mathrm{a}}$ \\
G2 & $65 \pm 11,8^{\mathrm{a}}$ & $69 \pm 13,9^{\mathrm{a}}$ & $65 \pm 5^{\mathrm{a}}$ & $67 \pm 9,7^{\mathrm{a}}$ & $39 \pm 16,8^{\mathrm{a}}$ & $54 \pm 8,6^{\mathrm{a}}$ \\
G3 & $38 \pm 9,6^{\mathrm{b}}$ & $34 \pm 8,3^{\mathrm{b}}$ & $62 \pm 10,6^{\mathrm{a}}$ & $60 \pm 5,2^{\mathrm{a}}$ & $39 \pm 6^{\mathrm{a}}$ & $35 \pm 11,9^{\mathrm{a}}$ \\
\hline
\end{tabular}

Média \pm desvio-padrão; a,b - Valores com sobrescritos diferentes na mesma coluna são estatisticamente diferentes de acordo com o teste de Student Newman Keuls $(\mathrm{p}<0,05)$, entre os meios diluidores; Não houve diferença significativa na motilidade entre temperaturas de descongelamento para os três meios testados. G1: Tris-cítrico-gema; G2: Lactose-gema; G3: INRA 82 ; A: $37^{\circ} \mathrm{C} / 30$ s; B: $52^{\circ} \mathrm{C} / 10 \mathrm{~s}$ seguido de $37^{\circ} \mathrm{C} / 30$ s. 
porém não obteve resultados satisfatórios na manutenção da motilidade e da morfologia espermática. Apesar da presença do leite desnatado e de carboidratos em sua composição, como glicose, lactose e rafinose [22], a quantidade de gema do ovo presente na formulação pode ter influenciado a taxa de motilidade espermática após o descongelamento. A proporção de gema do ovo indicada para meios de congelamento do sêmen canino é de 20\% [20]. Enquanto o papel protetor da gema é bem conhecido no processo de congelamento, ainda não está comprovado o mesmo efeito dos componentes do leite desnatado sobre a membrana espermática [3]. Uma pesquisa testando cinco meios de congelamento para o sêmen canino verificou que, apesar do leite desnatado apresentar características favoráveis à criopreservação de sêmen, como viscosidade, fonte energética e atividade antibacteriana, ele também pode causar alterações na morfologia espermática que se refletem na motilidade [18].

As temperaturas de descongelamento não influenciaram os resultados obtidos nas avaliações da motilidade espermática, indicando que para a curva de congelamento utilizada usando máquina computadorizada esse fator não exerceu influência para esse quesito após o descongelamento. A temperatura de descongelamento pode ser alterada de acordo com o meio diluidor e a curva de congelamento. Células congeladas rapidamente podem conter pequenos cristais de gelo que, se o descongelamento for realizado também rapidamente, geralmente não implica morte celular. Porém, se o descongelamento ocorrer lentamente, há uma reorganização dos cristais de gelo, que aumentam seu tamanho e lesionam as células [16]. A temperatura de $37^{\circ} \mathrm{C}$ por 30 segundos apresentou resultados favoráveis quanto à manutenção da morfologia e da integridade estrutural de membrana, sendo utilizada no descongelamento do sêmen canino [19,22]. Um autor afirma que o uso do protocolo de descongelamento à temperatura de $55 \% 10$ s para palhetas de $0,5 \mathrm{~mL}$ é capaz de obter resultados de motilidade espermática satisfatórios, porém ele não foi eficaz na preservação da morfologia normal do acrossoma e da peça intermediária no presente trabalho [5].

\section{CONCLUSÕES}

Com base nos resultados obtidos, pode-se concluir que os meios diluidores Tris-cítrico-gema e lactosegema foram mais eficientes na manutenção da viabilidade espermática para o protocolo de congelamento utilizado. O meio INRA 82 foi menos eficiente em preservar a motilidade espermática e a morfologia após o descongelamento em cães. A temperatura de descongelamento a $52^{\circ} \mathrm{C} / 10$ s seguido de $37^{\circ} \mathrm{C} / 30$ s causou maior porcentagem de defeitos acrossomais e da peça intermediária.

Agradecimentos. À Fapemig, pelo suporte financeiro, e à empresa Tetakon, pelo empréstimo da máquina de congelamento.

\section{REFERÊNCIAS}

1 Álamo D., Batista M., González F., Rodríguez N., Cruz G., Cabrera F. \& Gracia A. 2005. Cryopreservation of semen in the dog: use of ultra-freezers of $-152^{\circ} \mathrm{C}$ as a viable alternative to liquid nitrogen. Theriogenology. 63: 73-82.

2 Batista M., Álamo D., González M.G., Cruz M.G. \& Gracia A. 2006. Influence of the freezing technique (Nitrogen liquid vs ultrafreezes of $-152^{\circ} \mathrm{C}$ ) and male-to-male variation over the semen quality in Canarian Mastiff breed dogs. Reproduction in Domestic Animals. 41: 423-428.

3 Bergeron A. \& Manjunath P. 2006. New insights towards understanding the mechanisms of sperm protection by egg yolk and milk. Molecular Reproduction and Development. 73: 1338-1344.

4 Bueno R., Costa E.P., Guimarães J.D. \& Valentim F.M. 2001. Qualidade espermática do sêmen criopreservado de cães. II - Efeito do protocolo de resfriamento. Arquivo Brasileiro de Medicina Veterinária e Zootecnia. 53: 372-379.

5 Eilts B.E. 2005. Theoretical aspects of canine semen cryopreservation. Theriogenology. 64: 692-697.

6 Graham J.K. \& Mocé E. 2005. Fertility evaluation of frozen/thawed semen. Theriogenology. 64: 492-504.

7 Harrison R.A.P. \& Vickers S.E. 1990. Use of fluorescent probes to assess membrane integrity in mammalian spermatozoa. Journal of Reproduction and Fertility. 88:343-352.

8 Hori T., Odaka S., Oba H., Mizutani T., Kawakami E. \& Tsutsui T. 2006. Effects of liquid nitrogen vapor sensitization conditions on the quality of frozen-thawed dog spermatozoa. Journal of Veterinary Medicine and Science. 68: 1055-1061.

9 Kumi-Diaka J. 1993. Subjecting canine semen to the hypo-osmotic test. Theriogenology. 39: 1279-1289.

10 Martin J.C., Klug E. \& Günzel A.R. 1979. Centrifugation of stallion semen and its storage in large volume straws. Journal of Reproduction and Fertility. 27 (Suppl 1): 47-51. 
11 Nöthling J.O. \& Shuttleworth R. 2005. The effect of straw size, freezing rate and thawing rate upon post-thaw quality of dog semen. Theriogenology. 63: 1469-1480.

12 Nöthling J.O., Dolieslager S.M.J., Fillekes R. \& Colenbrander B. 2007. Thawing dog spermatozoa in just-boiled water: Submersion time and effect on sperm quality compared to thawing in water at $70^{\circ} \mathrm{C}$. Theriogenology. $68: 530-537$.

13 Oliveira E.C.S., Juliani G.C., Henry M. \& Marques Jr. A.P. 2003. Viabilidade in vitro do sêmen canino submetido a congelação com diferentes diluidores e crioprotetores. Revista Brasileira de Reprodução Animal. 27: 363-365.

14 Palmer E. 1984. Factors affecting stallion semen survival and fertility. In: Proceedings of the 10 International Congress on Animal Reproduction Artificial Insemination. v.3. (Illinois, EUA). p. 377-378.

15 Peña A.I. 1997. Supervivencia y fertilidad del semen canino sometido a congelacion-descongelacion. 329f. Lugo, Galícia. Tese (Doutorado em Veterinária) - Universidade de Santiago de Compostela.

16 Peña F.J., Núñez-Martínez I. \& Morán J.M. 2006. Semen technologies in dog breeding: an update. Reproduction in Domestic Animals. 41 (Suppl 2): 21-29.

17 Rodriguez-Gil J.E. 2006. Mammalian sperm energy resources management and survival during conservation in refrigeration. Reproduction in Domestic Animals. 41(Suppl 2): 11-20.

18 Santos S.E.C., Vannucchi C.I., Satzinger S., Assumpção M.E.O.D. \& Visintin J.A. 1999. Comparison of five extenders for canine semen freezing. Brazilian Journal of Veterinary Research and Animal Science. 36: 1-11. [Fonte: http://www.scielo.br/ scielo.php?script=sci_issuetoc\&pid=1413-959619990005\&lng=en\&nrm=iso].

19 Santos S.E.C., Vannucchi C.I., Satzinger S. \& Visintin J.A. 2001. Comparação de dois crioprotetores na congelação de sêmen de cães. Revista Brasileira de Reprodução Animal. 25: 472-473.

20 Silva A.R., Cardoso R.C.S. \& Silva L.D.M. 2000. Congelação de sêmen canino com diferentes concentrações de gema de ovo e glicerol em diluidores à base de tris e água de coco. Ciência Rural. 30: 1021-1025.

21 Vidament M., Yvon J.M., Couty I., Arnaud G., Nguekam-Feugang J., Noue P., Cottron S., Le Tellier A., Noel F., Palmer E. \& Magistrini M. 2001. Advances in cryoprservation of stallion semen in modified INRA 82. Animal Reproduction Science. 68: 201-218.

22 Yildiz C., Kaya A., Aksoy M. \& Tekeli T. 2000. Influence of sugar supplementation of the extender on motility, viability and acrosomal integrity of dog spermatozoa during freezing. Theriogenology. 54: 579-585.

23 Yu I., Songsasen N., Godke R.A. \& Leibo S.P. 2002. Differences among dogs in response of their spermatozoa to cryopreservation using various cooling and warming rates. Cryobiology. 44: 62-78.

24 Zúccari C.E.S.N. 1998. Efeito da criopreservação sobre a integridade estrutural da célula espermática eqüina. 121f. Botucatu, SP. Tese (Doutorado em Reprodução Animal) - Programa de Pós-graduação em Reprodução Animal, Universidade Estadual Paulista. 